\title{
Deleting Files in the Celeste Peer-to-Peer Storage System
}

\author{
Gal Badishi* $^{* \dagger} \quad$ Germano Caronni $^{\ddagger} \quad$ Idit Keidar $^{\dagger} \quad$ Raphael Rom $^{\ddagger} \quad$ Glenn Scott ${ }^{\ddagger}$ \\ ${ }^{\dagger}$ Department of Electrical Engineering, The Technion - Israel Institute of Technology. \\ ${ }^{\ddagger}$ Sun Microsystems Laboratories.
}

\begin{abstract}
Celeste is a robust peer-to-peer object store built on top of a distributed hash table (DHT). Celeste is a working system, developed by Sun Microsystems Laboratories. During the development of Celeste, we faced the challenge of complete object deletion, and moreover, of deleting "files" composed of several different objects. This important problem is not solved by merely deleting meta-data, as there are scenarios in which all file contents must be deleted, e.g., due to a court order. Complete file deletion in a realistic peer-topeer storage system has not been previously dealt with due to the intricacy of the problem - the system may experience high churn rates, nodes may crash or have intermittent connectivity, and the overlay network may become partitioned at times. We present an algorithm that eventually deletes all file content, data and meta-data, in the aforementioned complex scenarios. The algorithm is fully functional and has been successfully integrated into Celeste.
\end{abstract}

\section{Introduction}

Two different technologies have been developed in recent years: network storage systems and peer-to-peer networking. Network storage, exemplified by a variety of NAS and SAN products, is a result of the realization that stored data is sufficiently valuable that reliable and continuous access to it is mandatory. Peer-to-peer systems have evolved to create large distributed systems from small and unreliable components, overcoming the cost of providing extremely reliable units. It was only a matter of time before these technologies merge to create peer-to-peer based storage systems. Examples of such systems, each emphasizing a different aspect of data storage, are Farsite [1], OceanStore [5], Venti [9], Freenet [4], and Ivy [6].

Each of these systems contains an overlay network, typically constructed on top of a distributed hash table (DHT), providing routing and object location services to a storage

\footnotetext{
* Supported by the Israeli Ministry of Science.
}

management system. Most of these storage systems consider aspects of data replication, reliability, security, and storage maintenance, but almost none of them addresses data deletion directly. It is noteworthy that Plaxton et al. [8], in their seminal paper, do address data deletion at the DHT level. However, their system does not address more complex (and perhaps higher level) issues of security, trust, and access control, which we consider crucial. Moreover, their system is static, rendering deletion a much easier problem than in a dynamic system as considered herein.

One can identify three tiers of data deletion. The first tier is access-based deletion, in which data is not actually removed but access to it is made harder. File systems typically delete pointers to the data (sometimes by modifying the file's meta-data). Another approach is to use data encryption in which case data deletion amounts to destroying the relevant keys [7]. This is the easiest of all tiers and relies on the inability to ever access data without pointers to it or decrypt data without knowing the relevant keys. In some cases, access-based deletion may be insufficient, such as due to court orders. Company lawyers, such as Sun Microsystem's, often demand that a storage system will have the ability to completely delete the contents of a file, so as to comply with the judge's ruling.

The second tier of deletion is data obliteration, in which data itself, not just the access means to it, are completely removed from the system. It is a better approach to data deletion as it is independent of future technological advances. This tier does not necessarily replace the first tier, but rather augments it with much stronger deletion guarantees.

The third tier is data annihilation, in which all traces of the data are removed from all media on which it is stored. This tier is extremely costly to implement and is typically reserved to national security related data. In this paper, we deal with data obliteration, i.e., the second tier.

Robustly obliterating a file in a survivable peer-to-peer storage system is a real challenge. The main difficulty lies in the mere fact that the storage system is designed to be survivable and to provide availability even when nodes crash and links fail. To allow that, the system usually cuts a file 
into multiple objects, replicates all of them, and stores their copies on different nodes. To enable complete deletion, all of these chunks must be located and deleted. What is worse is that the storage system might try to maintain a minimum number of copies available, so as to guarantee availability. This stands in contrast to what the deletion algorithm wishes to do. Additionally, nodes may join or leave the system at arbitrary times, in an orderly fashion, or simply by crashing or becoming part of a distinct network partition. The objects these nodes hold might re-enter the system at unknown times in the future. By this time, the node at which the deletion request was initiated may be unavailable. The system should always know whether an object that has entered the system should in fact be deleted. Finally, secure deletion means that only authorized nodes should be allowed to delete a specific file.

We present a deletion (in the sense of data obliteration) algorithm designed to operate on top of typical DHTs. The algorithm was implemented in Celeste [3], which is a large scale, distributed, secure, mutable peer-to-peer storage system that does not assume continuous connectivity among its elements. Celeste was designed and implemented at Sun Microsystems Labs. Our deletion algorithm is founded on a separate authorization and authentication mechanism to allow deletion of stored objects. It is based on secure deletion tokens, one per object, that are necessary and sufficient to delete an object.

In Section 2, we describe the system in which our deletion algorithm operates, namely, a storage system, (Celeste in our case), running atop a DHT, as well as the cryptographic authorization and authentication mechanisms used. Section 3 presents our deletion algorithm. This section describes the algorithm abstractly, without linking it to a particular implementation.

In Section 4, we formally prove sufficient conditions for the algorithm's success. Roughly speaking, we prove that in a stable network partition $P$ that includes at least one copy of each of the meta-data objects describing a file $F$, if any node in $P$ tries to delete $F$ (at any point in time), then the algorithm guarantees complete deletion of the contents of all data and meta-data objects associated with $F$ in $P$. Moreover, when such a partition $P$ merges with another partition $P^{\prime}$, our algorithm ensures complete deletion of all data objects associated with $F$ in $P \cup P^{\prime}$.

In Section 5, we validate the effectiveness of the algorithm through simulations in typical settings. First, we present static simulations showing that in a stable partition, complete deletion is achieved promptly. These simulations validate the correctness proof of Section 4, and demonstrate the deletion time in stable situations, where eventual complete deletion is ensured. Moreover, the algorithm is scalable: as the underlying communication is based on a DHT overlay, deletion time increases like the routing time over a
DHT - logarithmically with the number of nodes. We further show that the deletion time increases linearly with the number of versions the file has, but decreases with the number of replicas, since each node holding a copy of a metadata object of a file participates in the deletion process. At the same time, a larger number of replicas increases the message load on the system. Secondly, we simulate the algorithm in dynamic settings, where nodes constantly crash and recover. These simulations enact scenarios that are not covered by the correctness proof of Section 4, which only considers eventually-stable settings. Nevertheless, in these simulations, complete file deletion is always successful. Moreover, the time it takes for a file to be completely obliterated from the system is proportional to the time it takes failed nodes holding the file's objects to recover.

Finally, Section 6 addresses some implementation issues that arise when implementing our algorithm in a real system, namely Celeste. Section 7 concludes.

\section{System Architecture}

We model our peer-to-peer system as a dynamic, intermittently connected, overlay network of nodes that may join or leave the system in an orderly fashion and can also crash unexpectedly. Additionally, the overlay network may become partitioned due to link failures. Crashed nodes may come back to life, retaining their previous state. In this paper we do not deal with nodes that transfer state information from other nodes. Such a state transfer will only facilitate the deletion process, and thus our results provide a lower bound on the algorithm's robustness.

Our system is composed of two layers, similar to Oceanstore $[5,11]$. The lower layer is a DHT that is used for locating, storing and retrieving objects. Any DHT can be used, as long as it provides the interface and semantics described below. Exemplar DHTs are Tapestry [14], Pastry [12], Chord [13], and CAN [10]. Above the DHT layer resides the Celeste layer [3]. Celeste provides a secure, survivable, mutable object store utilizing the DHT.

The Celeste layer. Each object in the DHT has a global universal identifier (GUID), e.g., a 256-bit number. A Celeste object is comprised of two parts, the data and the metadata. The meta-data contains information about the object, and is used both by the DHT and by Celeste. (Note that Celeste's meta-data is different from the filesystem's notion of a file meta-data, which is typically stored in a separate Celeste object). The integrity of objects in the DHT can be verified, and nodes do not store objects that fail an integrity check. With respect to verifiability, an object belongs to one of two categories: (1) A self-verifiable object is an object whose GUID is a hash of a mixture of its data section and its meta-data section. (2) A signed object is an object whose GUID is arbitrary, but its meta-data contains a digital signature that allows verification of the object's integrity. It is 
important to note that no two objects have the same GUID. Not even self-verifiable objects with an identical data section (their meta-data section is different).

General information about the file is saved as an object of a special type, called an AObject. Among others it includes the GUID of the latest version. Thus the GUID of the AObject (AGUID) is a lead to all the versions of the file. Each file update generates a new version containing information on that update. Each version is described by a special object called a VObject, whose GUID is called a VGUID. The AObject contains the VGUID of the latest version of the file, and each VObject contains the VGUID of the previous version of the file. Since the AObject is mutable but maintains the same GUID, it is a signed object. In contrast, VObjects are self-verifiable. We note here that having versions is very similar to having different files represent different updates to the same core file. In that respect, versioning is just a property of our system, and can be replaced by other means for file updates.

For simpler storage and management, the file's data contents are divided into blocks. Each block is stored as a separate self-verifiable object called a BObject, whose GUID is called a BGUID. Each VObject contains a list of all the BGUIDs that hold the data for that version of the file. The relations among these entities are depicted in Figure 1. Note that all Celeste objects are replicated in the DHT.

The DHT layer. The DHT layer provides the Celeste layer with primitives of object storage and retrieval. The DHT recognizes nodes and stored objects. Every object is assigned a root which is uniquely chosen among the live nodes in the system. The root is determined based on the object's GUID. (Nodes and objects have GUIDs drawn from the same space.) For example, the object's root can be the live node with the lowest GUID that is not lower than the object's GUID (if no such node exists, then the root is the node with the lowest GUID).

The root of an object is ultimately responsible to track the whereabouts of copies of the object. To that end the root of an object keeps soft-state information about the location of each copy of the object in the system, through the use of backpointers - a list of nodes that hold the object's copies (for efficiency, these backpointers may also be cached on other nodes).

A node's DHT layer provides its Celeste with the ability to send messages to Celeste layers on other reachable nodes, as well as with the following local functions:

- storeObject. Stores an object locally and sends a publish message to the object's root. The publish happens both immediately and periodically, as long as the object is stored. The publish message allows the root to maintain its soft-state tracking information, by saving a backpointer to the publisher. Backpointers expire after some time, to make sure only up-to-date infor- mation is saved. Thus, it is important for the publish rate to be sufficiently high to avoid unnecessary backpointer expiration in the root.

- retrieveObject. Utilizes a backpointer in the object's root or on the way to it, to fetch a copy of the object if one exists.

Problem definition. We tackle the problem of completely deleting file contents from a peer-to-peer storage system. The deletion entails removing all the versions of the file, their contents and their associated meta-data, limiting the operation to authorized entities. The challenge lies in securely obliterating all these data in a survivable, dynamic, failure-prone system that continuously strives to keep replicated objects available even when nodes crash and links fail.

Mechanisms for deletion. Our object deletion mechanism relies on a deletion token (DT) and its hashed versionthe deletion token hash (DTH). A deletion token may be any number, e.g., a random 256-bit number. A single deletion token is associated with every object in Celeste (but different objects may have the same deletion token). The object's deletion-token hash, i.e., the hash of the deletion token, is stored in the object's meta-data. Conversely, the object's deletion token is kept away from the object prior to deletion. Celeste considers a user authorized to delete an object if it presents the object's deletion token. A correct node agrees to delete an object only when supplied with a deletion token that hashes to the object's deletion-token hash stored in the object's meta-data. For efficiency reasons, in Celeste all objects belonging to the same file have the same deletiontoken. To provide for secure deletion, the file's deletion token is only known to entities that are allowed to delete the file. The aforementioned mechanisms for object integrity verification must include the deletion token hash.

A user that wishes to delete an object needs to expose the object's deletion token by supplying it to nodes that hold the object. Once a deletion token is exposed, it is no longer secret, and every node can use it to request other nodes to delete copies of the object (obviously containing the corresponding deletion-token hash).

\section{Deletion Algorithm}

This section discusses the algorithm for deleting a file. The algorithm is presented in pseudocode in Figures 2 and 3. Implementation issues are deferred to Section 6.

The deletion of a file starts at one or more (authorized) nodes and propagates to all the nodes that hold the file's objects throughout the system. To start the deletion sequence, a node invokes deleteFile with two parameters: the file's AGUID, and an appropriate exposed deletion token. To get the gears of the deletion process in motion, all that deleteFile does is create one copy of an AObject corresponding to that file with an exposed deletion token. Such a copy 


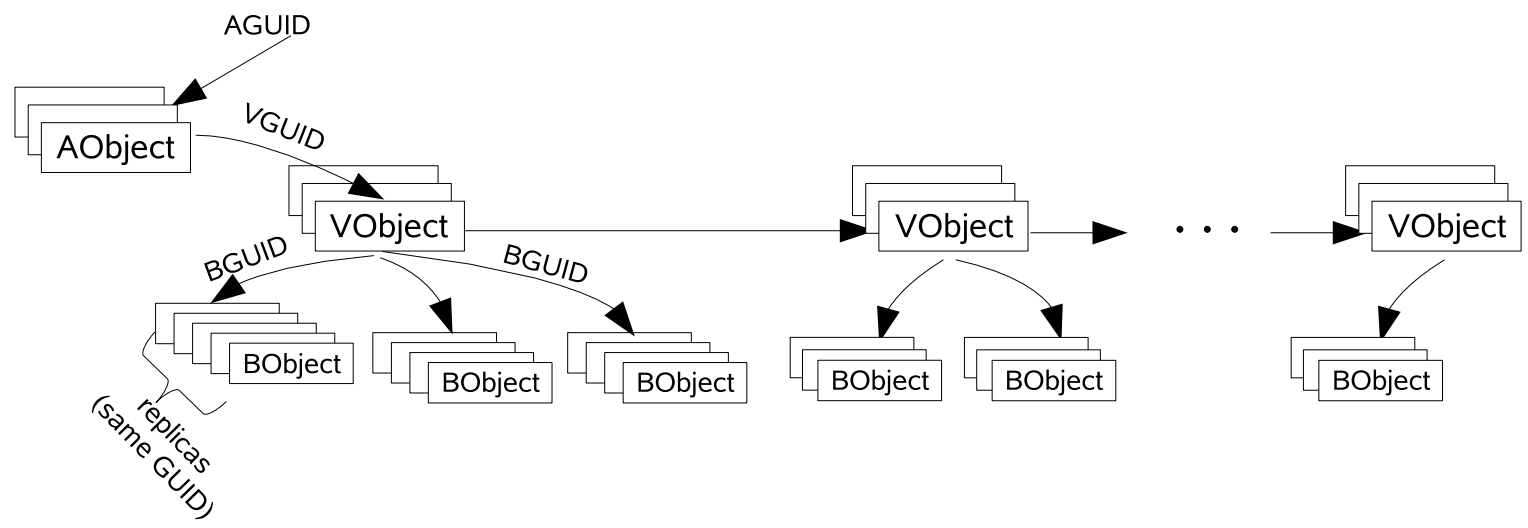

Figure 1. The relations between Celeste objects comprising a file.

is called a deletion object. A deletion object with GUID $\mathrm{g}$ serves as a permanent indication that the object whose GUID is $\mathrm{g}$ must be deleted. Creating such a deletion object does not necessitate fetching a copy of the original object; rather, an empty object with the same GUID and type and an exposed deletion token is created. After creating such an object, deleteFile invokes the procedure deleteObject for it.

Let us now overview the steps taken in the deletion of any object (cf. deleteObject, Figure 2). A node that begins the deletion process for an object $O$ is called a deleter of $O$. The deleter must have $O$ 's valid deletion token. If the deleter has already deleted $O$ in the past, (in which case it has a local copy of $O$ with an exposed deletion token), then it performs no further operations. As explained earlier, an object $O$ is deleted by storing a deletion object with $O$ 's GUID and an exposed deletion token. When an AObject or VObject is deleted, the deleter does some extra processing before storing this object, in order to ensure that the objects (VObjects and BObjects) that $O$ points to will also be deleted, as will be explained shortly.

After creating a deletion object $D_{O}$, the deleter calls the DHT's storeObject procedure in order to store a local copy of the deleted object. If a local copy of $O$ already exists, then storeObject replaces it by $D_{O}$, since $O$ and $D_{O}$ have the same GUID. In addition, storeObject issues a publish message, which winds its way to $O$ 's root, (again, since $O$ and $D_{O}$ have the same GUID, they have the same root). The deletion process is then continued by the root.

Thus, the mechanism of locally storing an object with an exposed deletion token serves as a persistent way to notify the object's root that the object should be deleted. Note that in a fault-prone dynamic system, an object's root may fail (crash) before completing the deletion, or even before being notified. But in this case, another node becomes the new root and since every Celeste replica periodically broadcasts a publish message toward the object's current root, the new root is eventually notified, and the deletion continues.

The root begins to engage in the deletion process upon

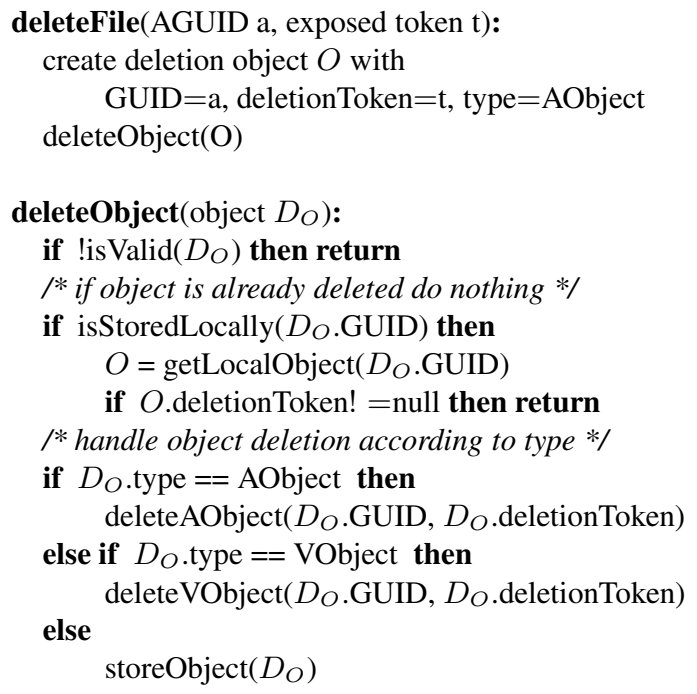

/*Deletion Procedure at the Object's Root */ Upon receiving publish message for object $O$ from $N$ : if $O$.deletionToken! =null then

if isDeleted(O.GUID) then return if ! is $\operatorname{Valid}(O)$ then return deleteObject $(O)$

for each backpointer! =me in backpointers(O.GUID) do send deletion request with $O$ to backpointer $\operatorname{markDeleted}(O$. GUID)

else $/ * O$ is not deleted $* /$

if isDeleted(O.GUID) then $D_{O}=$ getFromLocalStore(O.GUID) send deletion request for $D_{O}$ to $N$

Upon receiving deletion request with $D_{O}$ : if ! isStoredLocally $\left(D_{O}\right.$.GUID) then return deleteObject $\left(D_{O}\right)$

Figure 2. Basic deletion procedures at each node. 


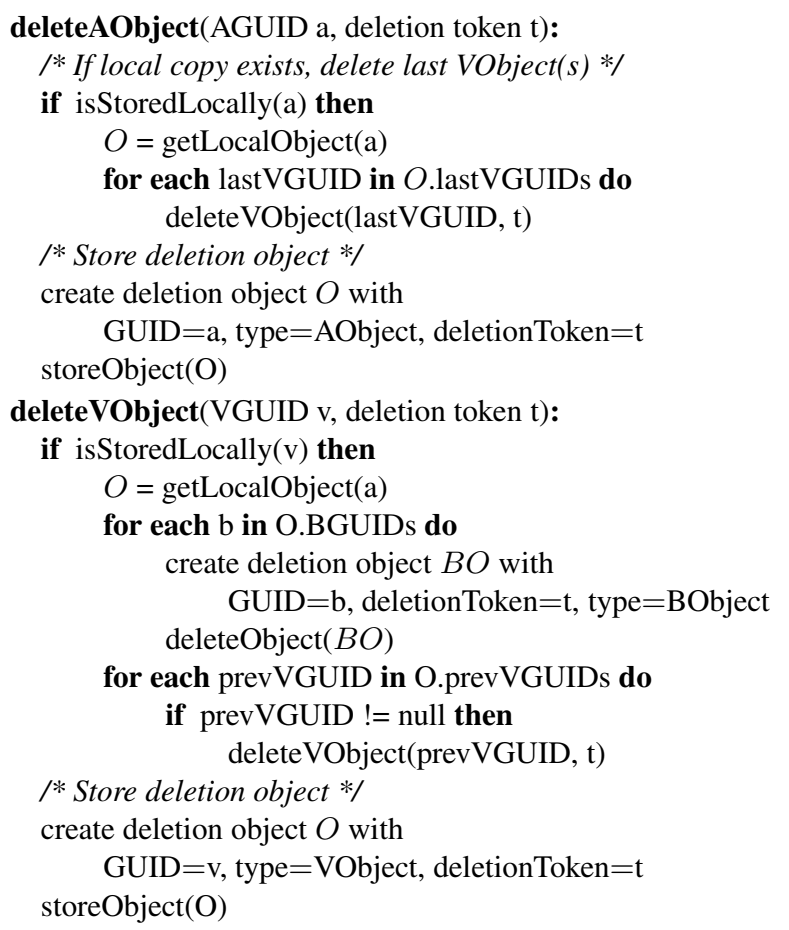

\section{Figure 3. Deletion procedures for AObjects and VObjects.}

receipt of a publish message for an object $O$ with an exposed deletion token. If the root has already deleted the object with $O$.GUID in the past, or if $O$ does not have a valid deletion token, then the root performs no further operations. The root deletes the object by calling the deleteObject procedure discussed above. The root then sends a deletion request message to each of the other nodes holding $O$, according to the known backpointers for $O$. The deletion request contains the published information for $O$, including the exposed deletion token and GUID. Finally, the root marks the deletion, that is, stores the information that $O$.GUID has been deleted. If the root subsequently receives a publish message for $O$, then it sends a deletion request to the node that sent the publish message.

Upon receiving a deletion request for $O$, the receiving node checks that is has $O$. If so, it deletes it using the deleteObject procedure.

AObjects are deleted in the procedure deleteAObject (see Figure 3 ). As before, the object is deleted by creating a deletion object with the object's GUID and an exposed deletion token, and storing it using the DHT's storeObject procedure. However, if the deleter holds a local copy of the object, then it cannot simply over-write it with a deletion one. This is because the AObject is the head of the linked list that contains all the file's VGUIDs, i.e., it is the head of the list that points to each version of the file. Thus, if the deleter has a local copy of the deleted AObject, it first initiates the deletion process for O.lastVGUID (by calling deleteVOb- ject), and only then deletes $O$ 's contents and stores it locally. Several "last" versions may exist due to partition merging.

VObjects are deleted in the procedure deleteVObject (see Figure 3). As before, the deleter creates a deletion object with the object's GUID and an exposed deletion token, and stores it. As with AObjects, before a node $N$ deletes a VObject $V_{i}$ from its local store, it must ensure that earlier versions will eventually also be deleted. First, $N$ deletes all the BObjects that are part of version $i$, by creating corresponding deletion objects and calling deleteObject for each of them. Then, $N$ checks whether an earlier version, $V_{i-1}$ was indeed created (several such versions may exist, due to merging). If so, it continues with the deletion process by calling deleteVObject for $V_{i}$. Finally, $N$ deletes the contents of the object and stores it with an exposed deletion token.

\section{Correctness}

In this section, we discuss the deletion algorithm's guarantees: we show sufficient conditions for complete deletion of a file. Although, as the simulations in the next section show, the algorithm succeeds in deleting files even in highly dynamic settings, it is very hard to reason about the algorithm in such settings. For the sake of reasoning about the algorithm, we examine stable periods. That is, we examine runs in which the system eventually stabilizes (the stabilization can occur either before or after the deletion initiation). Formally, stabilization is defined as follows:

Definition 1 (Stability). We say that partition P (consisting of a set of nodes) is stable in a time interval if in that interval all nodes can communicate with each other, nodes do not join or leave $P$, and no versions are added to files. We say that partition $P$ is eventually stable from time $T$ onwards if it is stable in $[T, \infty)$.

Note that in particular, nodes do not crash and crashed nodes do not recover in $P$ in a stable interval. We do not explicitly state the interval when it is clear from context.

In a partition $P$, our requirement from the algorithm is to completely delete the contents of all replicas of all objects pertaining to a deleted file. Formally:

Definition 2 (Deletion). An object copy is said to be deleted if its deletion token is exposed in its meta-data section and it has empty data contents. An object is deleted in partition $P$ if all its copies in $P$ are deleted. A file $F$ is deleted in $P$ if all objects pertaining to $F$ are deleted in $P$.

The dynamic failure-prone nature of the system renders the deletion problem (in the obliteration sense) unsolvable in some scenarios. Consider, for example, a file with two versions, and a stable partition $P$ in which no node holds a copy of last version's VObject. Since the only pointer to the first version is in that VObject, there is no way for the algorithm to discover the first version's objects, even 
if they do exist in $P$, and hence, there is no way to delete them. Therefore, in order to identify partitions where complete deletion is possible, we examine the nodes to which objects are assigned by Celeste when they are first stored, or at any time prior to the initiation of a deletion algorithm that should erase them:

Definition 3 (Assigned copies). A node $N$ is an assigned copy of object $O$ pertaining to a file $F$ if a copy of $O$ is stored at $N$ at a time $t$ so that deleteFile(F.GUID) is not invoked before time $t$.

This definition allows us to define a sufficient prerequisite for complete deletion of a file $F$ in a partition $P$ :

Definition 4 (Complete-deletion prerequisite for file $F$ ). We say that partition $P$ fulfills the complete-deletion prerequisite for file $F$, if F's AObject is assigned to some live node $N$ in $P, N$ has received the up-to-date version of $F$ 's AObject (pointing to the most recent version), and for each version that belongs to file $F$ there is a live node in $P$ to which the corresponding VObject is assigned.

We now turn to state some of the algorithm's guarantees. The proofs of the following theorem, as well as additional proven guarantees, are given in our full paper [2].

Theorem 1. Let $F$ be a file, and let AObj be the AObject representing $F$. If the following holds for partition $P$ : (1) $P$ is eventually stable from time $T$; (2) $P$ fulfills the completedeletion prerequisite for file $F$ at time $T$; and (3) A live node in $P$ holds a copy of the deleted AObj, $D_{A O b j}$, at time $T$, then all objects in $P$ associated with file $F$ are eventually deleted.

Corollary 1. Let F be a file, and let AObj be the AObject representing $F$. If the following holds for partition $P$ : (1) $P$ is eventually stable from time $T$; and (2) $P$ fulfills the complete-deletion prerequisites for $F$ at time $T$, then if a node $N$ in partition $P$ tries to delete file $F$ at any time $\tau$ (before or after $T$ ), all objects in $P$ associated with file $F$ are eventually deleted.

\section{Simulation Results}

Our deletion algorithm is implemented in Celeste, and was successfully tested in an environment containing a relatively small number of nodes. However, we would like to analyze the algorithm's expected behavior in actual deployment scenarios where many nodes are present. To test the effectiveness of our algorithm, we simulate it in diverse settings. The simulation is round-based. In each round, nodes perform whatever processing they need to do, and messages propagate at most one hop in the overlay network. Since the system is intended to be deployed in WANs, a round represents about 200 msecs. In each experiment, nodes publish their locally stored object copies every 20 seconds (100 rounds), and backpointers expire 100 seconds after receiving the last publish message. Each data point in each graph represents the average result of 500 experiments.

The system is composed of a single partition. In some experiments the partition is static, while in others nodes leave and join the partition by crashing and coming back to life, respectively. Messages sent between nodes are routed through intermediate nodes in the overlay. The number of hops it takes a message to reach its final destination is drawn from a bell-shaped distribution with a minimum of a single hop and a maximum logarithmic in the number of nodes. This latency distribution is typical in peer-to-peer overlays, and resembles one measured in Chord [13].

At the beginning of each experiment, a file is stored on random nodes. That is, all copies of each of the objects representing the file are stored on randomly-chosen nodes in the partition, such that no two copies of the same object are stored on the same node. After storing the file, an initial state, i.e., running or crashed, is selected for each node, according to the scenario that is being evaluated. After the nodes run for a while, a live node chosen uniformly at random attempts to delete the file.

We measure the time till complete deletion, i.e., the amount of time from the moment that some node initiates the deletion sequence, until all copies of objects associated with the file are deleted.

We do not consider nodes joining and leaving the partition, but rather nodes crashing and either staying down or coming back to life with their previous state intact. A node that comes back to life with its previous state, immediately publishes all of its objects and expires all of its stale backpointers. We note that this is a worst-case approach, which gives us an upper bound on the time till complete deletion. Obviously, the deletion time would not be higher had recovered nodes received state updates upon recovery.

We first evaluate our protocol in a static partition where all nodes remain up. This allows us to validate our simulation and provide insight on the impact of several parameters on the algorithm's performance. We then turn to examine our protocol in a dynamic setting, where nodes constantly crash and recover.

Failure-free operation. We first evaluate our deletion algorithm in a static, failure-free partition. Unless otherwise noted, the replication count is 5, and the file is represented by 1 AObject, 1 VObject, and 10 BObjects. I.e., the file has a single version with 10 data blocks, and there are 60 object copies stored in the DHT and associated with the file.

Figure 4(a) shows the time in rounds till complete deletion as a function of the number of nodes. Since the number of nodes is depicted on a logarithmic scale, we can see that the deletion time increases linearly. Due to this scalability, there is little importance in choosing a specific number of nodes to simulate. Henceforth, we simulate 1000 nodes. 


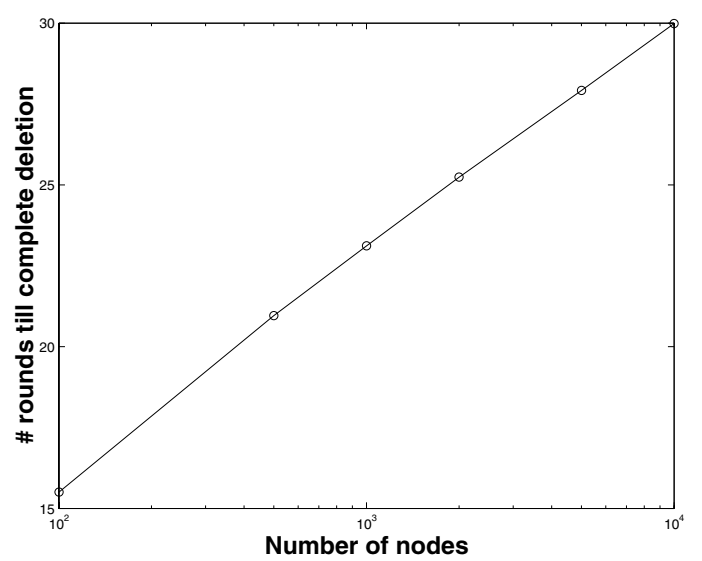

(a) Dependency on number of nodes (log), 1 version.

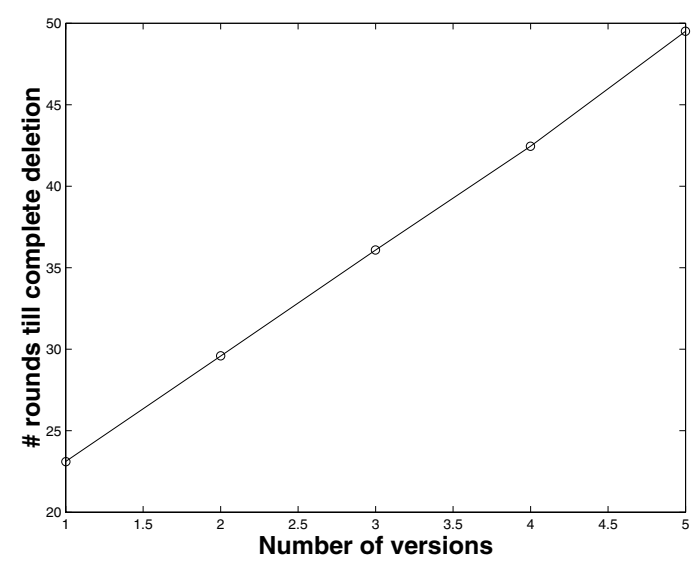

(b) Dependency on number of versions, 1000 nodes.

Figure 4. Average time till complete deletion, 10 blocks per version, 5 copies per object.

Figure 4(b) shows the time till complete deletion as a function of the number of versions. As expected, each additional version incurs the same overhead. Henceforth, we evaluate the algorithm for files with a single version. We have also measured the impact of increasing the number of of blocks (BObjects) per version, and found that it has a negligible effect on the time till complete deletion. (The graph is not shown here due to space considerations).

Figure 5(a) depicts the time in rounds till complete deletion as a function of the number of replicas per object. We can see that the more replicas there are, the faster the deletion completes. It is obvious that the deletion time should not increase as the number of copies increases, as all object copies are deleted roughly in parallel by simultaneous deletion requests sent from the object's root. The decrease in deletion time happens since having more copies per object increases the chance that some node holds copies of both the AObject and the VObject. Since the file has only a single version, all object copies associated with the file can be deleted in parallel when that node calls deleteAObject.

Figure 5(b) shows the number of messages in transit, i.e., the number of messages that have not yet reached their final destination. The peak at round 0 are all the publish messages sent by the nodes storing the object copies for the file. The number of publish messages is exactly the number of objects across nodes. For example, for a single version with replication factor of 5 we get 60 stored object copies. The number of messages quickly drops and stays low, as only publish messages are sent. The deletion is initiated at round 30 by a node chosen uniformly at random.

We see that the more versions we have, the longer it takes the system to become quiescent. This is due to the mostly-sequential nature of deleting several versions. We also see that when there are more replicas, more messages are sent per round. This expected behavior occurs since all backpointers are notified simultaneously, and all nodes storing a deleted object immediately publish it. The number of deleted objects is higher than the number of object copies, since nodes, e.g., roots, may hold deleted objects simply as an indication that the object is scheduled for deletion. This is the reason for the increase in the number of messages when the deletion completes, compared to the number of messages before the deletion starts - there are simply more objects to publish. Finally, the number of messages drops to 0 when the round number approaches 100 . This is simply an artifact of the experiments being shut down.

The results of this section validate Theorem 1 . We further note that when the assumptions of Theorem 1 hold in a partition, the time till complete deletion after the partition stabilizes (i.e., from time $T$ onwards) is no worse, and often better than the time till complete deletion measured in our failure-free evaluations. This is due to the smaller number of live nodes in the partition.

Dynamic simulations. We now simulate the algorithm in a partition that is never eventually stable. Each node is either crashed or running at any given time, and crashed nodes eventually run again, and vice versa. Node session times (time to failure) and down times (time to recovery) are distributed exponentially. When crashed nodes recover, they hold the same objects and backpointers they had when they crashed, and they immediately publish their stored objects and expire stale backpointers. We measure the time till complete deletion, that is, we must wait for crashed nodes holding parts of the file to recover before we can declare success. In all of our simulations, all of the files were eventually completely deleted. Thus, our algorithm achieves its goals under broader circumstances than formally established in the previous section.

We start all experiments in the partition's steady state, i.e., the node's initial state, up or down, is determined with respect to its uptime - the percentage of time in which the node is alive. Figure 6 shows the average time till complete deletion as a function of the nodes' uptime, where all nodes have the same uptime. Each node's average session time is 


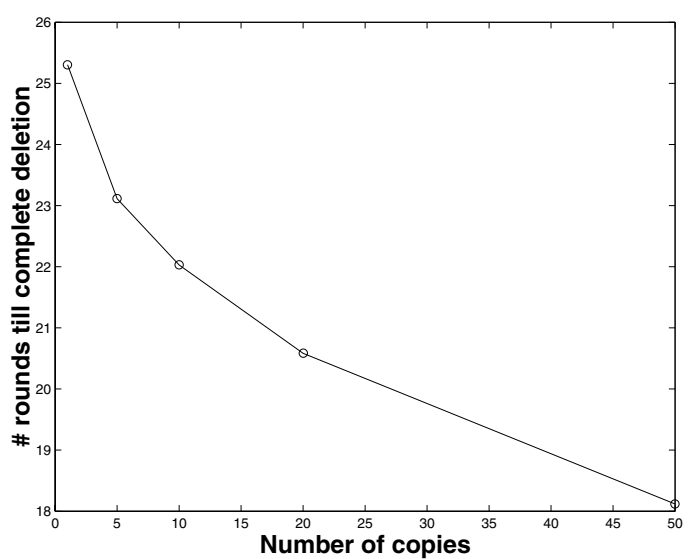

(a) Dependency on number of replicas, 1 version.

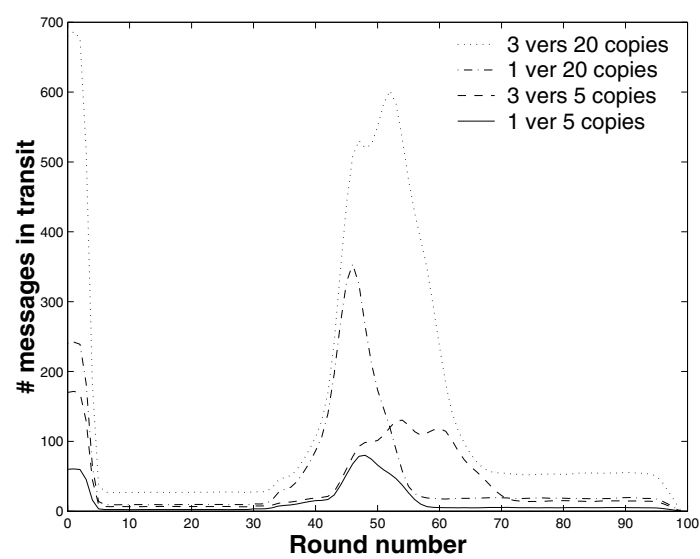

(b) Message overhead.

Figure 5. Average time till complete deletion and message overhead for 1000 nodes, 10 blocks per version.

1 hour, i.e., a node fails on average after one hour of operation. We observe that the higher the uptime, the faster the deletion process completes. This is expected, as there is a lower probability of a data object residing on a crashed node at deletion time. When an object resides on a crashed node, the deletion process cannot complete before that node recovers. Having understood the dependence on uptime, we fix it at $99 \%$ for our next experiments, and measure the effect of other parameters.

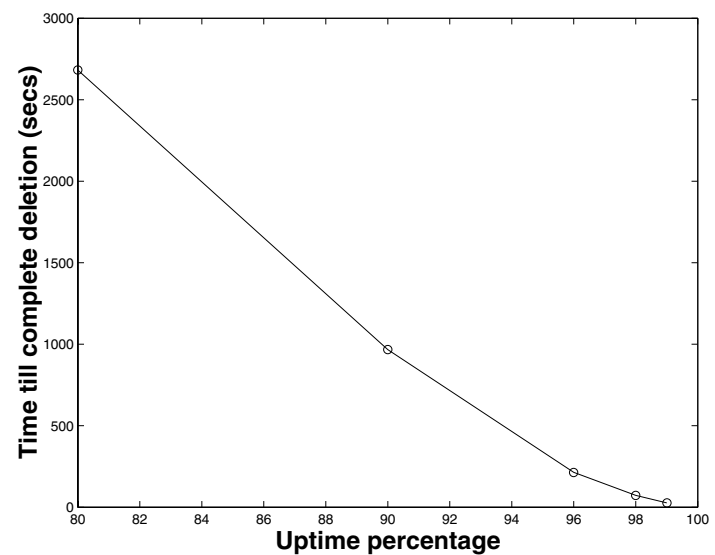

Figure 6. Average time till complete deletion vs. node uptime, 1000 nodes, 1 version, 10 blocks per version, 5 copies per object, average session time 1 hour.

Figure 7 shows CDFs of the time till complete deletion measured for 500 deletions. Figure 7(a) shows the dependency on the nodes' average session time. The longer the session time, the longer it takes to delete the file. Since the average uptime is fixed to $99 \%$, a longer session time means that if a node is down when the deletion request is issued, it will take the node longer to come back to life. This is exactly the time in which the data object is unreachable and cannot be deleted. Once the node comes back to life, the object it holds can be deleted promptly.

Figure 7(b) shows the how the number of object copies affects the time till complete deletion in a failure-prone system. We can see that fewer replicas mean better deletion times, in contrast to the result we get in a failure-free system, as depicted in Figure 5(a). The reason for this is that having more copies in a failure-prone system increases the probability that a crashed node has an object that needs to be deleted. The deletion process only completes when all such crashed nodes come back to life and receive deletion requests for their objects.

\section{Implementation Issues}

We have implemented the deletion algorithm presented in Section 3 in Celeste, a large-scale, peer-to-peer, secure mutable storage system developed in Sun Microsystems Labs. We now present some implementation specifics and discuss their impact on the deletion algorithm. Celeste is implemented in Java. It uses the hash function SHA-256 to generate GUIDs for nodes and objects.

Erasure coding. In addition to replication, Celeste supports erasure coding as a way of providing redundancy with reduced storage costs. In this case, a VObject points to fragments in addition to blocks. The fragments are used when the blocks are unavailable (unreachable). Fragment objects (FObjects) are treated by the deletion algorithm the same way as BObjects are. When an object is fragmented, the GUIDs of its fragments are stored alongside the original GUID itself (e.g., in the AObject or in another VObject). If the VObject fragments are redundant, i.e., a complete VObject exists as well, then they are treated like FObjects. Otherwise, before deleting an object that points to a fragmented VObject, the fragmented VObject is reconstructed 


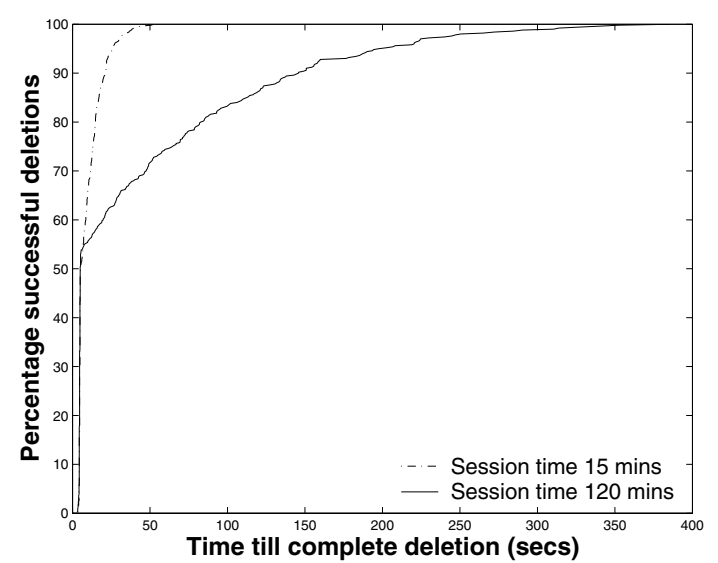

(a) Dependency on session time, 5 copies per object.

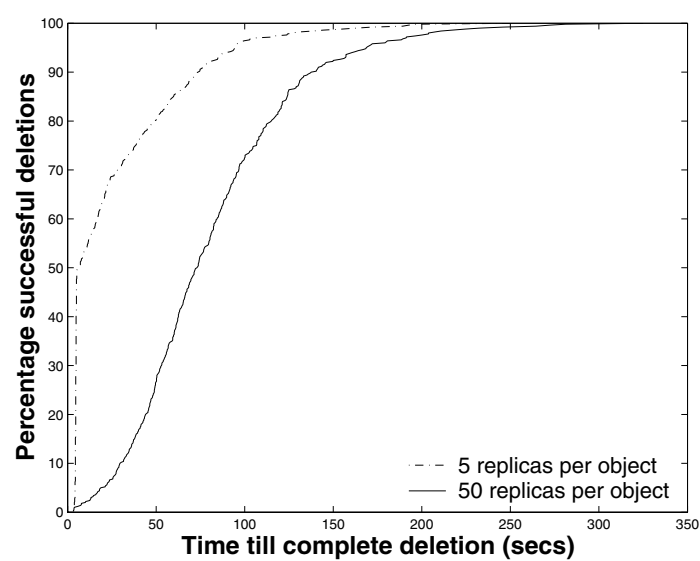

(b) Dependency on replication, 1 hour average session.

Figure 7. CDFs of $\mathbf{5 0 0}$ deletions showing dependency on session time and replication, 1000 nodes, 1 version, 10 blocks per version, $99 \%$ node uptime.

(by retrieving sufficiently many fragments), stored locally, and deleted using deleteVObject. Inability to reconstruct the VObject is equivalent to it being unreachable. In such cases, Celeste relies on application users to re-try deletion. In general, erasure coding makes the deletion process easier, since each fragment has a different GUID and is not replicated.

Refreshing mechanism. Since some nodes may permanently depart from the system, Celeste implements a refreshing mechanism to ensure that enough copies (or fragments) of a given object are available. To this end, it supports the role of a refresher, a node that creates new replicas when their number in the partition is too low. In order to work well with the deletion algorithm, we dictate that the refresher be the object's root. Thus, once the root is notified of a deletion it ceases to refresh the object.

Access rights. Celeste provides support for version access rights using encryption and digital signatures. For each version of a file, Celeste maintains a list of nodes that may either read, modify, or delete the version. When embarking on the deletion of a file, Celeste first deletes the list data used to access the file, effectively causing the file to be inaccessible. (Note that this is what we call tier one deletion).

Preventing over-writing of deleted objects. Recall that our deletion algorithm does not directly delete objects, but rather replaces them with empty objects that have the same GUID and the corresponding exposed deletion token in their meta-data. Once this has happened, it is important to ensure that the the deletion object will not be over-written by another (new) data object having the same GUID, since the correctness of our algorithm depends on the existence of such a deletion object. To this end, we have modified the DHT's storeObject function so that it does not allow overwriting an object with an exposed deletion token.

Protection against malicious attempts to prevent deletion. Although our algorithm is not intended for use with Byzantine nodes, Celeste takes extra measures to prevent malicious nodes from jeopardizing the system. One easy attack on the deletion algorithm can be realized by a malicious node $M$ as follows: $M$ retrieves an object $O$ that it does not want deleted (e.g., the AObject of a file that $M$ wishes to "protect"), and changes its deletion token hash. It then publishes the modified object, and causes correct nodes to store it. Subsequently, if $O$ is scheduled to be deleted, its modified version will not be deleted from correct nodes that hold it, since the correct deletion token cannot be validated against the bogus deletion-token hash. Thus, correct nodes will continue to store copies of $O$ permanently. In order to prevent such attacks, our implementation uses verifiable deletion tokens. It is important to note that this mechanism only overcomes attacks by nodes distinct from the object's root. If a malicious node succeeds in becoming an object's root, then it can still effectively prevent the deletion process from propagating to correct nodes that hold the object.

One way to realize verifiable deletion tokens in selfverifiable objects, where the object's GUID is the hash of its data contents, is to make sure the deletion-token hash is also part of the GUID. That is, GUID = $H($ data $\mid H($ deletiontoken $))$, where $H$ is a hash function, e.g., SHA-256, and | is the concatenation sign. Now, forging a deletion-token hash changes the GUID of the object.

However, since correct nodes only store objects they can validate, how can deletion objects be validated? In essence, a simple deletion object $D_{O}$ only carries the deletion token used for deletion, and has the same GUID as the object $O$ being deleted. If we have $O$, we can hash $D_{O}$ 's deletion token and compare it to $O$ 's deletion-token hash to verify $D_{O}$ 's validity. The problem is that the root $\operatorname{Root}_{O}$ of $O$ and $D_{O}$ receives the publish message for the first copy of $D_{O}$, and must verify $D_{O}$ before sending deletion requests to all the nodes holding copies of $O$. If $\operatorname{Root}_{O}$ did not previously 
receive any publish message for $O$, it cannot verify $D_{O}$. In that case, it can either save $D_{O}$ in a limited "unverified" list, or quietly discard it - it will be published again anyhow. If Root $_{O}$ does have some backpointer to $O$, then it knows $O$ 's deletion-token hash, as it is part of the meta-data sent in a publish message. Root $_{O}$ can then verify $D_{O}$ and continue.

But this solution assumes $\operatorname{Root}_{O}$ can trust the publish message for $O$. When Root $_{O}$ receives a publish message for $O$, it gets $O$ 's GUID, and the deletiontoken hash, but it does not receive $O$ 's data contents. Consequently, Root $_{O}$ cannot verify the binding $G U I D=H($ data $\mid H($ deletiontoken $))$ and must blindly trust the publisher. To allow the root to verify publish messages and deletion objects in all situations, self-verifiable GUIDs can be calculated as GUID = $H(H($ data $) \mid H($ deletiontoken $))$, and the meta-data portion of all objects, including deletion objects, should also include $H($ data $)$, i.e., the hash of the object's data contents. For a deletion object $D_{O}$, this is the hash of the deleted object $O$ 's data contents.

Finally, AObjects are mutable, and hence cannot be selfverifiable; they are verified using digital signatures. Naturally, the AObject's deletion-token hash should also be signed, so it cannot be fabricated.

Optimization for large files or small networks. When deleting a file, messages containing the deletion token are sent to all nodes involved in the deletion process. If the number of objects related to the file to be deleted exceeds some threshold in the order of $\frac{n}{\log n}$, where $n$ is the total number of nodes in the system, a simple broadcast of the deletion token may be better in terms of network load, and perhaps even latency. The threshold is based on the expected propagation time (in hops) in the overlay network.

Garbage collection. To free space consumed by deletion objects, it is possible to set an expiration time for every object in the system, including those that are not deleted. All objects belonging to the same file have the same expiration time. Nodes can safely garbage collect (remove) an object from their local store if that object's expiration time has passed. For every object associated with a file, the object's expiration time can only be set and modified by the file's creator, and only if the file has not been deleted yet. The refresher maintains sufficient object copies in the system only if the relevant file's expiration time has not passed yet. Thus, deleting a file with an expiration time set ensures that eventually all objects of that file will be garbage collected. However, since all state is soft, removing all deletion objects for a file will allow malicious nodes to reintroduce the deleted file to the system after the expiration time passes.

\section{Conclusions}

We have presented an algorithm for complete deletion of files (tier two deletion - including data obliteration) in a peer-to-peer storage system. The complete deletion of a file in such a system is an intricate operation, as it involves deleting multiple replicas of many (data and meta-data) objects, which may not all be available at the same time.

We have rigorously proven our algorithm's correctness in eventually stable runs. Moreover, we have demonstrated, using simulations, that the algorithm always succeeds to delete multi-object files entirely, even in highly dynamic fault-prone environments. Finally, we have discussed some practical issues that arose when implementing the algorithm in Celeste, a working peer-to-peer storage system developed at Sun Microsystems labs.

\section{References}

[1] A. Adya, W. J. Bolosky, M. Castro, G. Cermak, R. Chaiken, J. R. Douceur, J. Howell, J. R. Lorch, M. Theimer, and R. P. Wattenhofer. FARSITE: Federated, available, and reliable storage for an incompletely trusted environment. In $O S D I$, Dec 2002.

[2] G. Badishi, G. Caronni, I. Keidar, R. Rom, and G. Scott. Deleting files in the Celeste peer-to-peer storage system. TR CCIT 594, Department of EE, Technion, Jul 2007.

[3] G. Caronni, R. Rom, and G. Scott. Maintaining object ordering in a shared $\mathrm{p} 2 \mathrm{p}$ storage environment. In 3rd International IEEE Security in Storage Workshop, Dec 2005.

[4] I. Clarke, S. Miller, T. Hong, O. Sandberg, and B. Wiley. Protecting freedom of information online with Freenet. IEEE Internet Computing, Jan-Feb 2002.

[5] J. Kubiatowicz, D. Bindel, Y. Chen, S. Czerwinski, P. Eaton, D. Geels, R. Gummadi, S. Rhea, H. Weatherspoon, W. Weimer, C. Wells, and B. Zhao. OceanStore: An architecture for global-scale persistent storage. In ASPLOS, Nov 2000.

[6] A. Muthitacharoen, R. Morris, T. M. Gil, and B. Chen. Ivy: A read/write peer-to-peer file system. In OSDI, Dec 2002.

[7] Z. Peterson, R. Burns, J. Herring, A. Stubblefield, and A. Rubin. Secure deletion for a versioning file system. In FAST, San Francisco, Dec 2005.

[8] C. G. Plaxton, R. Rajaraman, and A. W. Richa. Accessing nearby copies of replicated objects in a distributed environment. TOCS, 32, 1999.

[9] S. Quinlan and S. Dorward. Venti: a new approach to archival storage. In FAST, 2002.

[10] S. Ratnasamy, P. Francis, M. Handley, R. Karp, and S. Shenker. A scalable content-addressable network. In Proceedings of ACM SIGCOMM, 2001.

[11] S. Rhea, P. Eaton, D. Geels, H. Weatherspoon, B. Zhao, and J. Kubiatowicz. Pond: the OceanStore prototype. In FAST, Mar 2003.

[12] A. Rowstron and P. Druschel. Pastry: Scalable, distributed object location and routing for large-scale peer-to-peer systems. In IFIP/ACM International Conference on Distributed Systems Platforms (Middleware), pages 329-350, Nov 2001.

[13] I. Stoica, R. Morris, D. Liben-Nowell, D. R. Karger, M. F. Kaashoek, F. Dabek, and H. Balakrishnan. Chord: A scalable peer-to-peer lookup protocol for Internet applications. IEEE/ACM Transactions on Networking. To Appear.

[14] B. Y. Zhao, L. Huang, J. Stribling, S. C. Rhea, A. D. Joseph, and J. D. Kubiatowicz. Tapestry: A resilient global-scale overlay for service deployment. IEEE JSAC, 22(1), Jan 2004. 